\title{
Pig Pluripotent Stem Cells as a Candidate for Biomedical Application
}

\author{
Kwang-Hwan Choi ${ }^{1}$ and Chang-Kyu Lee ${ }^{1,2, *}$ \\ ${ }^{1}$ Department of Agricultural Biotechnology, Animal Biotechnology Major, and Research Institute of Agriculture and Life \\ Science, Seoul National University, Seoul 08826, Korea \\ ${ }^{2}$ Institute of Green Bio Science and Technology, Seoul National University, Pyeongchang 25354, Korea
}

Received September 10, 2019

Revised September 23, 2019

Accepted September 26, 2019

*Correspondence

Chang-Kyu Lee

E-mail: leeck@snu.ac.kr

ORCID

https://orcid.org/0000-0001-6341-0013

\begin{abstract}
Stem cells are progenitor cells that are capable of self-renewal and differentiation into various cells. Especially, pluripotent stem cells (PSCs) have in vivo and in vitro differentiation capacity into three germ layers and can proliferate infinitely. The differentiation ability of PSCs can be applied for regenerative medicine and tissue engineering. In domestic animals, their PSCs have a potential for preclinical therapy as well as the production of transgenic animals and agricultural usage such as cultured meat. Among several domestic animals, a pig is considered as an ideal model for biomedical and agricultural purposes mentioned above. In this reason, studies for pig PSCs including embryonic stem cells (ESCs), embryonic germ cells (EGCs) and induced pluripotent stem cells (iPSCs) have been conducted for decades. Therefore, this review will discuss the history of PSCs derived from various origins and recent progress in pig PSC research field.
\end{abstract}

Keywords: embryonic germ cells, embryonic stem cells, induced pluripotent stem cells, pig, pluripotent stem cells

\section{INTRODUCTION}

Stem cells are progenitor cells that are capable of selfrenewal and differentiation into several cells. Especially, pluripotent stem cells (PSCs) have in vivo and in vitro differentiation potentials into three germ layers and can proliferate infinitely. First PSCs, known as embryonic stem cells (ESCs), were derived from preimplantation mouse blastocysts (Evans and Kaufman, 1981; Martin, 1981). Subsequently, another pluripotent cell lines, called embryonic germ cells (EGCs), were obtained through in vitro-culture of primordial germ cells (PGCs) (Matsui et al., 1992; Resnick et al., 1992). Recently, mouse epiblast stem cells (EpiSCs) and induced pluripotent stem cells (iPSCs) were derived from postimplantation embryos and somatic cells, respectively (Takahashi and Yamanaka, 2006; Tesar et al., 2007). Pluripotent states are divided into "naive" and "primed" states depending on the developmental competence of PSCs (Nichols and Smith, 2009). Naive PSCs, represented by mouse ESCs and EGCs, have developmental ground state similar with early epiblasts of preimplantation embryos. On the other hand, primed PSCs, including EpiSCs and human ESCs, possess more differentiated pluripotency than naïve cells showing features of late epiblasts in postimplantation embryos. In permissive lines, both states of PSCs can be derived from embryos. However, in non-permissive lines such as human, only primed PSCs are derived in the absence of additional treatment such as genetic manipulation and chemical supplementation (Nichols and Smith, 2009). 
Because PSCs not only can differentiate into various type of cells and tissues but also can produce germlinechimera by blastocyst injection (Bradley et al., 1984), PSCs have been considered as cell sources for cell therapy and transgenic animal production. After the establishment of human ESCs (Thomson et al., 1998), various researches for regenerative medicine by tissue engineering have been studied. Degenerative diseases could be treated by replacement of damaged tissues or cells with undamaged normal tissues or cells differentiated from PSCs. And the establishment of induced pluripotent stem cells (iPSCs) and cloned human ESCs allowed patient-specific cell therapies with PSCs (Takahashi and Yamanaka, 2006; Tachibana et al., 2013). In domestic animals, stem cell research aims to create an indefinite cell source for transgenic animals used as bioreactors and tissue engineering materials as well as preliminary studies for human research. To apply human PSCs as the tools for regenerative medicine, preclinical studies with animal models are essential. Several animals such as pig, horse, cow, and sheep, etc. have been used for PSC researches (Ogorevc et al., 2016). Especially, pigs have been identified as an ideal animal model to study human disease, because of the physiological and anatomical similarities of organs between humans and pigs (Park et al., 2013). So, in this review, it will be discussed that characteristics of PSCs derived from various origins and recent progress in pig PSC research area.

\section{BEGINNING OF PLURIPOTENT CELL RESEARCH}

Researches on pluripotent stem cells (PSCs) were begun with embryonal carcinoma cells (EC cells) derived from murine gonadal teratomas and teratocarcinoma. Teratoma (benign) and teratocarcinoma (malignant), as the tumors which are spontaneously formed in the gonad, are composed of several adult tissues including tooth, bone, muscle, skin, and hair, etc. When the cells from teratocarcinoma were intraperitoneally injected into strain 129 mice, they re-formed teratocarcinoma (Kleinsmith and Pierce, 1964). These findings showed that stem cell population which can proliferate indefinitely having a differentiation potential into several tissues existed in teratocarcinomas. In subsequent research, teratocarcinomas were artificially generated by injecting pre- and post-implantation em- bryos into the testis in mouse, which indicated that the stem cells in teratocarcinoma were similar with undifferentiated early embryos (Stevens, 1970). For the teratocarcinoma researches, Stevens developed 129/sv strain which has increased formation rate of the gonadal tumor (Stevens, 1981).

In the 1970s, Martin and Evans succeeded in isolating stem cells from teratocarcinoma, so-called EC cells, by co-culture with feeder cells made of mitotically-inactivated mouse embryonic fibroblasts (Martin and Evans, 1974). Subsequently, many scientists had analyzed the characteristics of EC cells, which paved the way for researches on PSCs. They reported that EC cells possessed differentiation ability into three germ layers, so-called pluripotency, and could proliferate indefinitely through symmetric division, so-called self-renewal. Similar to teratocarcinoma, when subcutaneously injected into a mouse, EC cells generated teratocarcinoma which has various differentiated tissues. When cultured in suspension, the cells aggregated and formed an embryo-like structure with a cavity, known as cystic embryoid bodies (Martin and Evans, 1975). In 1977, human EC cells were derived from malignant testicular teratomas and immunostaining showed that mouse and human EC cells expressed SSEA1 and SSEA4, respectively (Hogan et al., 1977). Based on their features recapitulating embryonic development, early PSC researches had focused on cellular differentiation and embryology.

Although EC cells were considered as the potential materials to study pluripotent cells and embryogenesis, they have limitations to study embryology. They had abnormal karyotype and, when micro-injected into recipient blastocysts, couldn't generate germline-chimeras, which means they possess restricted differentiation ability (Blelloch et al., 2004). In 1981, independent two groups solved the problems through direct seeding of early blastocyst onto feeder cells not using subcutaneous transplantation into a mouse and eventually established embryonic stem cells (ESCs) (Evans and Kaufman, 1981; Martin, 1981). ESCs not only possessed similar feature with EC cells in terms of cellular physiology, pluripotency and marker expression but also could produce germline-chimeras with normal karyotype via blastocyst injection (Bradley et al., 1984). Especially, as gene targeting technologies were developed, a transgenic mouse could be generated from genetically modified ESCs, which accelerated studies on gene functions during embryogenesis (Thomas and Capecchi, 
1986). On the other hand, because teratocarcinoma is spontaneously derived from germ cells in the testis, several research groups had tried to establish pluripotent cell lines from germ cells like what ESCs were derived from embryos. In 1992, these efforts resulted in establishing novel pluripotent cell lines, known as embryonic germ cells (EGCs) from primordial germ cells (PGCs) (Matsui et al., 1992; Resnick et al., 1992).

In 1998, PSC researches entered a new phase by the establishment of human ESCs (Thomson et al., 1998). The derived human ESCs made people think that pluripotent cells could be used for regenerative medicine by tissue engineering. To curing degenerative diseases including Parkinson's disease, Alzheimer's disease, diabetes, and retinitis pigmentosa, etc., damaged tissues or cells should be replaced with undamaged normal tissues or cells, but it is hard to prepare the sufficient amount of cells to care patients. However, if PSCs can be differentiated into specific cell lineage, human ESCs could supply sufficient amount of normal cells or tissues for transplantation. For these reasons, PSC researches started to focus on cell therapy and were accelerated by the establishment of induced pluripotent stem cells (iPSCs) and cloned human ESCs in 2006 and 2013, respectively (Takahashi and Yamanaka, 2006; Tachibana et al., 2013).

\section{EMBRYONIC STEM CELLS (ESCs)}

As mentioned above, embryonic stem cells (ESCs) are pluripotent cells derived from preimplantation embryos. To retain characteristics of ESCs such as self-renewal and pluripotency, various extrinsic and intrinsic factors have to be delicately regulated. Moreover, distinct features of ESCs compared with somatic cells are used to identify them. Lack of extrinsic or intrinsic determinants induces loss of pluripotency and differentiation of ESCs. So, it is important to understand and investigate genes and culture conditions involved in the pluripotency network circuit.

The microenvironmental surrounding of ESCs, known as a stem cell niche, including cytokines, extracellular matrix, and metabolic sources is important extrinsic factors for supporting pluripotency. Viability and selfrenewal of ESCs during in vitro-culture are dependent on the stem cell niche. Firstly derived mouse ESCs were obtained by co-culturing with mitotically-inactivated mouse embryonic fibroblasts (MEFs), so-called feeder layer, and
EC cell-derived conditioned media (Evans and Kaufman, 1981; Martin, 1981). The main function of the feeder layer is providing a physical environment such as the extracellular matrix (ECM), which affects proliferation and survival rate by activating Cadherin and Integrin signaling in ESCs (Guilak et al., 2009). And feeder cells activate intrinsic pathways of pluripotency by paracrine effects of several cytokines including LIF, FGF2, BMP4, ACTIVIN A, and WNT, etc (Eiselleova et al., 2008). Recently, to reduce cell-to-cell variation and xeno-contamination occurred by feeder layer, a mixture of ECM proteins and synthetic peptides are being used instead of feeder cells.

Together with the physical environment, activation of cellular signaling by cytokines is also important to maintain pluripotency. Because signaling molecules specifically support pluripotency of ESCs were not known, EC cell-derived conditioned media was used at the beginning of ESC culture (Martin, 1981). Later, it was verified that LIF and BMP4 signaling pathways have a crucial role in supporting the pluripotency of ESCs (Ying et al., 2003). Although LIF treatment is sufficient to maintain pluripotency in serum-supplemented media, ESCs differentiated into neural lineage in serum-free media, suggesting unknown factors of fetal bovine serum involved in sustaining pluripotency. Ying and colleagues found that BMP4 supports pluripotency with LIF in serum-free media preventing neural differentiation (Ying et al., 2003). BMP4 facilitates expression of inhibitor of differentiation, Id, protein through SMAD signaling and these proteins inhibit neural differentiation. Feeder cells are also required for culture of human ESCs, similar to the mouse (Thomson et al., 1998). However, there are some physiological differences between human and mouse ESCs. Unlikely mouse in which LIF and BMP4 are involved, the pluripotency of human ESCs is sustained through ERK and ACTIVIN/NODAL signal pathway activated by FGF2 and TGF- $\beta$ (Pera and Tam, 2010). These distinguishable features had been considered as species-specific characteristics for a long time. However, series of experiments discovered that the differences among PSCs are originated by two distinct grades of pluripotency, including "naïve" and "primed" states, based on developmental competence (Nichols and Smith, 2009).

\section{EPIBLAST STEM CELLS (EpiSCs)}

In 2007, two research groups established a novel type of 
pluripotent stem cells, named postimplantation epiblastderived stem cells (shortly, epiblast stem cells or EpiSCs), from postimplantation embryos (Brons et al., 2007; Tesar et al., 2007). To derive EpiSCs, late epiblasts were isolated at embryonic day 5.5 before gastrulation and seeded onto feeder cells in culture media supplemented with FGF2 and ACTIVIN A. After 3-5 days, epiblast explants rapidly grew and formed OCT4-expressed compact colonies. The established EpiSCs expressed pluripotent genes such as Oct4, Sox 2 , and Nanog, and were capable of generating teratomas when the cells were grafted in immune-deficiency mice. However, EpiSCs showed distinct characteristics compared with ESCs derived from early epiblasts. Expression of ICM-specific genes which are up-regulated in ESCs such as Pecam1, Tbx3, and Gbx2 was decreased in EpiSCs. On the other hand, epiblast and early germ layer-specific genes such as Otx2, Eomes, Foxa2, T, Gata4, Sox17 and Cer1 were highly expressed in EpiSCs. This cell line grew as a monolayer having flattened morphology and relied on ERK and ACTIVIN/NODAL signaling pathway to maintain pluripotency instead of LIF and BMP4 signaling. In epigenetic and developmental aspects, female EpiSCs possessed inactivated X chromosome similar to their in vivo-counterpart, late epiblasts. In the same manner, when assessed developmental stage by chimeric assay, although they could not generate chimeric embryos with morula and early blastocyst, EpiSCs were incorporated within embryos and developed chimeric fetus when engrafted into postimplantation embryos (Kojima et al., 2014). While murine ESCs could be converted into EpiSCs by treatment of FGF2 and LIF antagonist, EpiSC couldn' $\mathrm{t}$ be converted into ESCs without genetic manipulations, which means steps of mammalian development are irreversible (Nichols and Smith, 2009). Overall, EpiSCs shared the similarities with human ESCs in terms of gene expression and cellular signaling.

\section{EMBRYONIC GERM CELLS (EGCS)}

PGCs as an alternative cell source can be used for deriving pluripotent stem cells. When cultured with feeder cells and adequate cytokines, PGCs can be reprogrammed into pluripotent stem cells, named EGCs. As ESCs were established from ICM via in vitro-culture, it had been attempted that in vitro culture of PGCs, the origin of spontaneous teratocarcinoma in vivo, for deriving novel pluripotent stem cell line. When cultured with feeder cells made of STO cell line and cytokines including LIF, FGF2, and SCF, mouse migrating PGCs isolated at dpc 8.5 were reprogrammed and converted into pluripotent EGCs (Matsui et al., 1992; Resnick et al., 1992). Although PGCs undergo apoptosis in a few days during in vitro culture, Stem cell factor (SCF, also known as c-Kit ligand or Steel factor) prevented apoptosis and promoted viability and proliferation rate of germ cells together with LIF (Matsui et al., 1991). FGF2 reportedly up-regulates the expression of Dhx38, Myc and Klf4 known as targets of BLIMP1 via down-regulation of Blimp1, facilitates reprogramming of PGCs into EGCs (Durcova-Hills et al., 2008). Finally, fullyreprogrammed mouse EGCs cultured with only LIF. Established mouse EGCs possess similar features with ESCs in physiological and developmental aspects. They express pluripotent marker genes such as OCT4, SOX2, NANOG and SSEA1, and have developmental competence in vitro (embryoid body formation) as well as in vivo (teratoma and chimera formation). Besides, recent data indicated that there are close similarities between EGCs and ESCs in terms of genetics and epigenetics (Choi et al., 2017). In human, EGCs could be derived from the gonad of aborted fetuses culturing with feeder cells and LIF, FGF2, and Fsk (Shamblott et al., 1998). Human EGCs expressed several pluripotent markers such as OCT4, SSEA1, and SSEA3/4 and have in vitro developmental competence. However, because of ethical restrictions for using human fetuses, research on human germ cells has not been deeply performed.

\section{INDUCED PLURIPOTENT STEM CELLS (iPSCS)}

In 2006, Yamanaka and colleagues developed a new method to easily reprogram somatic cells to pluripotent cells (Takahashi and Yamanaka, 2006). Newly derived PSCs, so-called iPSCs, were generated from adult somatic cells by nuclear reprogramming via ectopic expression of four genes such as Oct4, Sox2, Klf4, and cMyc (generally called 'Yamanaka's factors'). Comparison of global gene expression between ESCs and iPSCs showed that the gene expression pattern of two cell lines was very similar. The cells could stably be maintained having a normal karyotype and differentiate into three germ layers in vitro and in vivo. In 2007, human iPSCs were derived by two independent groups (Takahashi et al., 2007; Yu et al., 2007). 
Yamanaka and colleagues generated human iPSCs by Yamanaka's factors, the other groups used different combinations including OCT4, SOX2, NANOG, and LIN28. The iPSC technique was much easier to use compared with prior dedifferentiation methods (SCNT and cell fusion) and could be applied to adult and aged cells, which accelerated stem cell researches for regenerative medicine and cell therapy using patient-specific PSCs.

However, iPSC technique has problems for applying to therapeutic usages. First of all, abnormal features could be occurred by the integration of transgenes in the genome of cells during the maintenance and differentiation of iPSCs. Especially, one of Yamanaka's factors, cMyc as a proto-oncogene, induced tumor formation when iPSC/ iPSC-derived cells were transplanted in vivo (Nakagawa et al., 2010). And also, because epigenetic memory derived from the origin of somatic cells were remained in iPSCs after reprogramming, differentiation of iPSCs were biasedly progressed (Polo et al., 2010). And this deficiency of epigenetic reprogramming caused differences between ESCs and iPSCs (Chin et al., 2010). These problems were solved with integration-free gene delivery systems such as plasmid vector, episomal vector, piggyback transposon system, adenovirus vector and sendai virus vector, and transgene-free methods such as protein- and chemicalmediated systems. And a recent study showed that such variations between ESCs and iPSCs were mainly originated by different genetic background and genetically matched human ESCs and iPSCs were molecularly and functionally equivalent (Choi et al., 2015).

\section{LARGE ANIMAL MODELS IN STEM CELL BIOLOGY: A PIG REVIEW}

Pig has a great potential in xenotransplantation and disease model for human and in biopharming, because of its anatomical and physiological similarities with human (Park et al., 2013). PSCs of domestic animals have been identified as promising tools for generating transgenic animals and preclinical researches. Several studies showed that ESCs were more efficient for cloning of mouse than differentiated cells such as immune cells, neuron, and fibroblasts (Hochedlinger and Jaenisch, 2002). And PSCs have another advantage in application to nuclear transfer. PSCs can proliferate indefinitely maintaining cellular characteristics including karyotype and genomic stabil- ity. This property, so-called self-renewal, provides an indefinite cell source for nuclear transfer. With genetic manipulations, they can offer stable transgenic cell source for the generation of transgenic animals for bioreactors, xenotransplantation and disease models. And also, as PSCs could be incorporated in the development of early embryos, blastocyst injection could be applied for a generation of transgenic animals. In 2014, the first clinical test was operated in Japan (Sugita et al., 2016). A woman who suffered from aged-macular degeneration (AMD) received retinal pigment epithelium (RPE) differentiated from iPSCs, and her vision was partially recovered. However, because spinal cords and eyes are an immune-privileged area without immune cells and are not affected to life, clinical tests for organs and tissues which are strongly involved in life, such as heart, brain, and pancreas, need strict preclinical test using animal disease models. So, it makes the porcine study involving differentiation and transplantation of stem cells as a preliminary study more important.

In early studies of pig embryonic stem cells (ESCs), researchers tried to establish stem cells based on teratocarcinoma culture methods as mouse studies did. Various culture materials including serums (fetal bovine serum and calf serum), feeder cells (buffalo liver cells, mouse embryonic fibroblasts, pig embryonic fibroblasts, and pig uterine epithelial cells) and conditioned media were tested for maintaining pluripotent inner cell mass (ICM) in vitro (Anderson et al., 1994). Nonetheless, it was hard to derive pig stem cells due to a lack of knowledge about cell signaling involved in early embryo development and maintaining pluripotency. In addition, many cytokines like interleukins (IL), oncostatin M (OSM), ciliary neurotrophic factor (CNTF), epidermal growth factor (EGF), activin A (ActA) and stem cell factor (SCF) as well as LIF and FGF2 have been used for in vitro culture of pig ICM with various combinations and concentrations. However, the in vitro-cultured ICM lost their own features and in turn differentiated during prolonged culture. Instead, during in vitro-culture, multipotent stem cells, so-called ES-like cells, have been spontaneously derived by several groups (Park et al., 2013). Established pig ES-like cell lines have shown some common features. They express pluripotent markers such as AP, OCT4, SOX2, and NANOG, and have in vitro-differentiation ability, but not in vivo-developmental competence (chimera and teratoma formation 
ability).

Because molecular biological differences existed during embryo development among mouse, human, and pig, the culture conditions for human and mouse ESCs have not been able to support pig pluripotency in vitro. During the development of the early embryo, which has a pluripotent inner cell mass (ICM), the pig has a longer preimplantation period than that of mouse and human. In order to overcome these hurdles, Choi and Lee et al. systemically examined effects of various culture conditions on pig pluripotency, and finally developed the pig-specific ESC culture media containing FGF2, Activin A, and inhibitors of GSK3 and canonical WNT pathway. Unlike previous reports, the cells derived by novel culture media can maintain over 50 passages and differentiate into three germ layers in vitro and in vivo. They were capable of direct differentiation into specific cells including neural cells, pancreatic progenitor and cardiac muscle and transcriptomically similar with human PSCs than mouse PSCs, which indicates that these cells could have the potential for preclinical study of human cell therapy (Choi et al., 2019). And they found lipid supplementation was crucial for maintaining pig pluripotency in vitro, which indicated that metabolic niche also affects deriving and maintaining pluripotency in the ungulate.

Because it has proven difficult to obtain authentic pig ESCs, various research groups were focusing on somatic cell reprogramming in a pig. Induced pluripotent stem cell (iPSC) lines in pigs were firstly generated by independent three groups in 2009 (Esteban et al., 2009; Ezashi et al., 2009; Wu et al., 2009). These cell lines were derived from fetal fibroblasts by using viral vectors carrying different reprogramming factors (human OSKM (Ezashi et al., 2009), mouse OSKM (Esteban et al., 2009), and human OSKM + NANOG and LIN28 (Wu et al., 2009)). Interestingly, pig iPSC lines have primed pluripotent state in terms of morphologic and molecular features resembling mouse EpiSCs and human ESCs, rather than mouse ESCs. In addition, in LIF and small molecules (especially ERK and GSK signaling inhibitors)-supplemented media, naive-like pig iPS cell lines have been obtained. The pig naïve iPSC lines showed expression of naïve-pluripotent markers, two active $\mathrm{X}$ chromosomes, and differentiation potential into three germ layers (Fujishiro et al., 2013). However, the majority of pig iPSC lines were partially reprogrammed, showing transgene dependency and in- complete epigenetic reprogramming. Several studies demonstrated that reprogramming of pig cells has unique characteristics governed by different cellular networks compared with that of mouse and human (Choi et al., 2016). To derive fully-reprogrammed pig iPSCs, it is required to develop pig-specific culture system and reprogramming methods along with identifying pig pluripotent markers based on developmental biology.

Pig germ-line stem cells have been studied for a long time. Porcine primordial germ cells (PGCs) arise in endoderm and mesoderm beside stalk of the yolk sac and allantois during gastrulation (embryonic day (E) 13-14). Pig PGCs are starting to migrate from endoderm and mesoderm and arrive at genital ridges in E22 via dorsal mesentery of the hindgut. At around E30, primitive gonads develop into sex organs (Hyldig et al., 2011). Global DNA demethylation involving repetitive elements as well as erasing gender-specific imprints occur during migration. And gender-specific imprinting patterns are reestablished between E25-31 (Hyldig et al., 2011). Unlike mouse study, pig embryonic germ cells (EGCs) have been isolated from E25-30 gonadal PGCs, while pig migrating PGCs couldn't be converted into EGCs (Petkov et al., 2011). It might be caused by differences in the timing of epigenetic changes between mouse and pig PGCs (Hyldig et al., 2011). In agreement with mouse PGCs, pig PGCs are converted into EGCs by FGF2-mediated reprogramming (Choi et al., 2018). When FGF2 was withdrawn during primary culture of PGCs, the number of primary EGC colonies was dramatically reduced (Lee and Piedrahita, 2000). Along with FGF2, membrane-bound/soluble SCF and LIF have pivotal roles in survival and proliferation of pig PGCs during primary culture. However, long-term culture in media containing LIF caused down-regulation of pluripotency genes and FGF2 supplement was more efficient for maintaining the pluripotency in pig EGCs (Choi et al., 2018). Without SCF, in vitro-survival rate of PGCs were reduced and, during extended culture, EGC colonies disappeared (Lee and Piedrahita, 2000).

\section{PERSPECTIVES}

Overall, the advancement of stem cell techniques mentioned above will help to improve human welfare in the aspect of healthy life and agricultural production via generation of high-production and disease-resistance 
Choi and Lee. Pig Pluripotent Stem Cells

animals. To accomplish these goals with pig PSCs, there are hurdles remain to be overcame. First of all, although recent study represented authentic pig ESCs can derive using chemically defined media (Choi et al., 2019), it is required to develop more defined culture conditions such as feeder-free culture and reprogramming method for pig PSCs. Analysis of enhancer showed that different transcription factors were involved in the expression of OCT4 known as a core gene of pluripotency networks (Kim et al., 2019), which means a majority of pig pluripotency circuit remains to be elucidated. And to create genetically engineered animals by using pig PSCs, genetic manipulation via transgenic technologies has been required in stem cell research. As vectors carrying transgenes, plasmid vector, virus vectors (including lentiviral vector, retroviral vector, sendai virus vector and adenovirus vector), episomal vector and piggyback transposon system have been developed. If transfected with short hairpin RNA (shRNA)expressing vector, expression of a targeted gene can be reduced instead of upregulation. In addition to inserting transgenes, replacement and disruption of endogenous genes can be accomplished by gene targeting using homologous recombination, known as a repair mechanism of DNA double-strand. Finally, as a preclinical trial of human PSCs for cell therapy, the research of animal PSCs involving differentiation and transplantation study are important. In this reason, many researchers have been attempted to convert the porcine PSCs into several differentiated cells including neural, and rod photoreceptors. More diverse type of cells would be derived from pig PSCs and various transplantation models are going to develop in the near future. Overall, pig pluripotent stem cells have tremendous potentials for preclinical study, comparative developmental biology, and transgenic animal production. These efforts may lead to the success of cell therapy and improvement of agricultural production.

\section{CONFLICTS OF INTEREST}

No potential conflict of interest relevant to this article was reported.

\section{ACKNOWLEDGEMENTS}

This work was supported by the BK21 Plus Program and the National Research Foundation of Korea (NRF) grant fund- ed by the Korea government (NRF-2019R1C1C1004514).

\section{ORCID}

Kwang-Hwan Choi: https://orcid.org/0000-0003-3919-7413

Chang-Kyu Lee: https://orcid.org/0000-0001-6341-0013

\section{REFERENCES}

Anderson GB, Choi SJ, Bondurant RH. 1994. Survival of porcine inner cell masses in culture and after injection into blastocysts. Theriogenology. 42:204-212.

Blelloch RH, Hochedlinger K, Yamada Y, Brennan C, Kim M, Mintz B, Chin L, Jaenisch R. 2004. Nuclear cloning of embryonal carcinoma cells. Proceedings of the National Academy of Sciences of the United States of America. 101:1398513990.

Bradley A, Evans M, Kaufman MH, Robertson E. 1984. Formation of germ-line chimaeras from embryo-derived teratocarcinoma cell lines. Nature. 309:255-256.

Brons IG, Smithers LE, Trotter MW, Rugg-Gunn P, Sun B, Chuva de Sousa Lopes SM, Howlett SK, Clarkson A, AhrlundRichter L, Pedersen RA, et al. 2007. Derivation of pluripotent epiblast stem cells from mammalian embryos. Nature. 448: 191-195.

Chin MH, Pellegrini M, Plath K, Lowry WE. 2010. Molecular analyses of human induced pluripotent stem cells and embryonic stem cells. Cell Stem Cell. 7:263-269.

Choi J, Clement K, Huebner AJ, Webster J, Rose CM, Brumbaugh J, Walsh RM, Lee S, Savol A, Etchegaray JP, et al. 2017. DUSP9 Modulates DNA Hypomethylation in Female Mouse Pluripotent Stem Cells. Cell Stem Cell. 20:706-719.

Choi J, Lee S, Mallard W, Clement K, Tagliazucchi GM, Lim H, Choi IY, Ferrari F, Tsankov AM, Pop R, et al. 2015. A comparison of genetically matched cell lines reveals the equivalence of human iPSCs and ESCs. Nat Biotechnol. 33:1173-1181.

Choi KH, Lee DK, Kim SW, Woo SH, Kim DY, Lee CK. 2019. Chemically Defined Media Can Maintain Pig Pluripotency Network In Vitro. Stem Cell Reports. 13:221-234.

Choi KH, Lee DK, Oh JN, Son HY, Lee CK. 2018. FGF2 Signaling Plays an Important Role in Maintaining Pluripotent State of Pig Embryonic Germ Cells. Cell Reprogram. 20:301-311.

Choi KH, Park JK, Son D, Hwang JY, Lee DK, Ka H, Park J, Lee CK. 2016. Reactivation of Endogenous Genes and Epigenetic Remodeling Are Barriers for Generating Transgene-Free Induced Pluripotent Stem Cells in Pig. PLoS One. 11:e0158046.

Durcova-Hills G, Tang F, Doody G, Tooze R, Surani MA. 2008. Reprogramming primordial germ cells into pluripotent stem cells. PloS one. 3:e3531.

Eiselleova L, Peterkova I, Neradil J, Slaninova I, Hampl A, Dvorak P. 2008. Comparative study of mouse and human feeder cells for human embryonic stem cells. The International 
Journal of Developmental Biology. 52:353-363.

Esteban MA, Xu J, Yang J, Peng M, Qin D, Li W, Jiang Z, Chen J, Deng K, Zhong M, et al. 2009. Generation of induced pluripotent stem cell lines from Tibetan miniature pig. The Journal of Biological Chemistry. 284:17634-17640.

Evans MJ, Kaufman MH. 1981. Establishment in culture of pluripotential cells from mouse embryos. Nature. 292:154-156.

Ezashi T, Telugu BP, Alexenko AP, Sachdev S, Sinha S, Roberts RM. 2009. Derivation of induced pluripotent stem cells from pig somatic cells. Proceedings of the National Academy of Sciences of the United States of America. 106:10993-10998.

Fujishiro SH, Nakano K, Mizukami Y, Azami T, Arai Y, Matsunari H, Ishino R, Nishimura T, Watanabe $\mathrm{M}$, Abe T, et al. 2013. Generation of naive-like porcine-induced pluripotent stem cells capable of contributing to embryonic and fetal development. Stem Cells Dev. 22:473-482.

Guilak F, Cohen DM, Estes BT, Gimble JM, Liedtke W, Chen CS. 2009. Control of stem cell fate by physical interactions with the extracellular matrix. Cell Stem Cell. 5:17-26.

Hochedlinger K, Jaenisch R. 2002. Monoclonal mice generated by nuclear transfer from mature $\mathrm{B}$ and $\mathrm{T}$ donor cells. Nature. 415:1035-1038.

Hogan B, Fellous M, Avner P, Jacob F. 1977. Isolation of a human teratoma cell line which expresses F9 antigen. Nature. 270:515-518.

Hyldig SM, Croxall N, Contreras DA, Thomsen PD, Alberio R. 2011. Epigenetic reprogramming in the porcine germ line. BMC Dev Biol. 11:11.

Kim SH, Choi KH, Lee DK, Lee M, Hwang JY, Lee CK. 2019. Identification and Characterization of the OCT4 Upstream Regulatory Region in Sus scrofa. Stem Cells Int. 2019: 2130973.

Kleinsmith LJ, Pierce GB Jr. 1964. Multipotentiality of Single Embryonal Carcinoma Cells. Cancer Research. 24:15441551.

Kojima Y, Kaufman-Francis K, Studdert JB, Steiner KA, Power MD, Loebel DA, Jones V, Hor A, de Alencastro G, Logan GJ, et al. 2014. The transcriptional and functional properties of mouse epiblast stem cells resemble the anterior primitive streak. Cell Stem Cell. 14:107-120.

Lee CK, Piedrahita JA. 2000. Effects of growth factors and feeder cells on porcine primordial germ cells in vitro. Cloning. 2: 197-205.

Martin GR. 1981. Isolation of a pluripotent cell line from early mouse embryos cultured in medium conditioned by teratocarcinoma stem cells. Proceedings of the National Academy of Sciences of the United States of America. 78:7634-7638.

Martin GR, Evans MJ. 1974. The morphology and growth of a pluripotent teratocarcinoma cell line and its derivatives in tissue culture. Cell 2:163-172.

Martin GR, Evans MJ. 1975. Multiple differentiation of clonal teratocarcinoma stem cells following embryoid body formation in vitro. Cell. 6:467-474.

Matsui Y, Toksoz D, Nishikawa S, Nishikawa S, Williams D, Zsebo K, Hogan BL. 1991. Effect of Steel factor and leukaemia inhibitory factor on murine primordial germ cells in culture. Nature. 353:750-752.

Matsui Y, Zsebo K, Hogan BL. 1992. Derivation of pluripotential embryonic stem cells from murine primordial germ cells in culture. Cell. 70:841-847.

Nakagawa M, Takizawa N, Narita M, Ichisaka T, Yamanaka S. 2010. Promotion of direct reprogramming by transformation-deficient Myc. Proceedings of the National Academy of Sciences of the United States of America. 107:14152-14157.

Nichols J, Smith A. 2009. Naive and primed pluripotent states. Cell Stem Cell. 4:487-492.

Ogorevc J, Orehek S, Dovc P. 2016. Cellular reprogramming in farm animals: an overview of iPSC generation in the mammalian farm animal species. J Anim Sci Biotechnol. 7:10.

Park JK, Kim HS, Uh KJ, Choi KH, Kim HM, Lee T, Yang BC, Kim $\mathrm{HJ}$, Ka HH, Kim H, et al. 2013. Primed pluripotent cell lines derived from various embryonic origins and somatic cells in pig. PLoS One. 8:e52481.

Pera MF, Tam PP. 2010. Extrinsic regulation of pluripotent stem cells. Nature. 465:713-720.

Petkov SG, Marks H, Klein T, Garcia RS, Gao Y, Stunnenberg H, Hyttel P. 2011. In vitro culture and characterization of putative porcine embryonic germ cells derived from domestic breeds and Yucatan mini pig embryos at Days 20-24 of gestation. Stem Cell Res. 6:226-237.

Polo JM, Liu S, Figueroa ME, Kulalert W, Eminli S, Tan KY, Apostolou E, Stadtfeld M, Li Y, Shioda T, et al. 2010. Cell type of origin influences the molecular and functional properties of mouse induced pluripotent stem cells. Nat Biotechnol. 28:848-855.

Resnick JL, Bixler LS, Cheng L, Donovan PJ. 1992. Long-term proliferation of mouse primordial germ cells in culture. Nature. 359:550-551.

Shamblott MJ, Axelman J, Wang S, Bugg EM, Littlefield JW, Donovan PJ, Blumenthal PD, Huggins GR, Gearhart JD. 1998. Derivation of pluripotent stem cells from cultured human primordial germ cells. Proceedings of the National Academy of Sciences of the United States of America. 95: 13726-13731.

Stevens LC. 1970. The development of transplantable teratocarcinomas from intratesticular grafts of pre- and postimplantation mouse embryos. Developmental Biology. 21:364-382.

Stevens LC. 1981. Genetic influences on teratocarcinogenesis and parthenogenesis. Progress in Clinical and Biological Research. 45:93-104.

Sugita S, Iwasaki Y, Makabe K, Kamao H, Mandai M, Shiina T, Ogasawara K, Hirami Y, Kurimoto Y, Takahashi M. 2016. Successful Transplantation of Retinal Pigment Epithelial Cells from MHC Homozygote iPSCs in MHC-Matched Models. Stem Cell Reports. 7:635-648.

Tachibana M, Amato P, Sparman M, Gutierrez NM, TippnerHedges R, Ma H, Kang E, Fulati A, Lee HS, Sritanaudomchai $\mathrm{H}$, et al. 2013. Human embryonic stem cells derived by somatic cell nuclear transfer. Cell. 153:1228-1238.

Takahashi K, Tanabe K, Ohnuki M, Narita M, Ichisaka T, Tomo- 
da K, Yamanaka S. 2007. Induction of pluripotent stem cells from adult human fibroblasts by defined factors. Cell. 131: 861-872.

Takahashi K, Yamanaka S. 2006. Induction of pluripotent stem cells from mouse embryonic and adult fibroblast cultures by defined factors. Cell. 126:663-676.

Tesar PJ, Chenoweth JG, Brook FA, Davies TJ, Evans EP, Mack DL, Gardner RL, McKay RD. 2007. New cell lines from mouse epiblast share defining features with human embryonic stem cells. Nature. 448:196-199.

Thomas KR, Capecchi MR. 1986. Introduction of homologous DNA sequences into mammalian cells induces mutations in the cognate gene. Nature. 324:34-38.

Thomson JA, Itskovitz-Eldor J, Shapiro SS, Waknitz MA, Swier- giel JJ, Marshall VS, Jones JM. 1998. Embryonic stem cell lines derived from human blastocysts. Science. 282:11451147.

Wu Z, Chen J, Ren J, Bao L, Liao J, Cui C, Rao L, Li H, Gu Y, Dai H, et al. 2009. Generation of pig induced pluripotent stem cells with a drug-inducible system. J Mol Cell Biol. 1:46-54.

Ying QL, Nichols J, Chambers I, Smith A. 2003. BMP induction of Id proteins suppresses differentiation and sustains embryonic stem cell self-renewal in collaboration with STAT3. Cell. 115:281-292.

Yu J, Vodyanik MA, Smuga-Otto K, Antosiewicz-Bourget J, Frane JL, Tian S, Nie J, Jonsdottir GA, Ruotti V, Stewart R, et al. 2007. Induced pluripotent stem cell lines derived from human somatic cells. Science. 318:1917-1920. 